\title{
Measured distribution of cloud chamber tracks from radioactive decay: a new empirical approach to investigating the quantum measurement problem
}

Jonathan Schonfeld ( $\sim$ jschonfeld@cfa.harvard.edu )

https://orcid.org/0000-0002-8909-2401

\section{Research Article}

Keywords: quantum measurement, quantum mechanics, cloud chamber

Posted Date: October 25th, 2021

DOI: https://doi.org/10.21203/rs.3.rs-892640/v3

License: (c) (i) This work is licensed under a Creative Commons Attribution 4.0 International License.

Read Full License 
Measured distribution of cloud chamber tracks from radioactive decay: a new empirical approach to investigating the quantum measurement problem

Jonathan F. Schonfeld

Center for Astrophysics | Harvard and Smithsonian

60 Garden St., Cambridge, Massachusetts 02420 USA

jschonfeld@cfa.harvard.edu

ORCID ID\# 0000-0002-8909-2401 
Abstract Using publically available video of a cloud chamber with a very small radioactive source, I measure the spatial distribution of where tracks start, and consider possible implications. This is directly relevant to the quantum measurement problem and its possible resolution, and appears never to have been done before. The raw data are relatively uncontrolled, leading to caveats that should guide future, more tailored experiments. Results suggest a modification to Born's rule at very small wavefunction. Track distributions from decays in cloud chambers represent a previously unappreciated way to probe the foundations of quantum mechanics, and a novel case of wavefunctions with macroscopic signatures.

Keywords Quantum measurement; quantum mechanics; cloud chamber

\section{Introduction}

Measurement occupies a privileged role in the conventional formulation of quantum theory. Between measurements, a wave function evolves smoothly according to Schroedinger's equation; but measurement itself is widely supposed to entail moments when the wavefunction changes discontinuously by application of random projections. This juxtaposition of smooth and discontinuous is the quantum measurement problem [1]. A solution to the problem could take the form of a demonstration that instantaneous projections are really idealized "effective representations" of more complicated processes governed entirely by smooth Schroedinger evolution. Such a solution would expose limits to projection-based formulations. Such limits could be consequential for quantum computing, in which quantum measurement is carried out on industrial scale; and for searches for events, such as proton decay, whose extreme rarity could be uncharted territory for canonical measurement phenomenology.

Quantum computing and proton-decay searches employ very sophisticated measurement technology. Since 2020 I've focused my own quantum-measurement research on the lower-tech cloud chamber, because its underlying physics is much simpler. In a recent paper [2], I explored the processes by which a cloud chamber detects single charged particles emitted by the simplest radioactive decays. I identified a mechanism to explain the origination of cloud chamber tracks without appeal to random projections, and derived an emergent position-space Born rule that describes the distribution in space and time of tracks' starting points. Deviations from the Born rule would presumably result when droplet formation in supersaturated vapors deviates from conventional idealizations. The Born distribution in Reference [2] depends on position in a very simple way, and should be no harder to verify experimentally than the familiar double-slit interference pattern, but I have been unable to find applicable experiments on the statistics of cloud-chamber track locations. So I have conducted a search on the internet for videos of cloudchamber tracks induced by radioactive decay, and have measured a track distribution myself in one video to compare with Reference [2]. The purpose of this paper is to report my methodology and findings.

As will be apparent, it is premature to draw firm conclusions from comparison between theory and measurement, but the results do suggest a modification to Born's rule at very small wavefunction. Caveats arise from many uncontrolled aspects of the underlying data. Issues include: uncontrolled temperature and vapor density in the cloud chamber; uncalibrated placement 
of the video camera; suboptimal and varying placement of the illumination source; and indeterminate thermal characteristics of the sample's mounting fixture. These caveats are all good justifications for building an apparatus tailored to the purpose at hand, instrumented to reveal and diagnose deviations between measurement and theory. Judged against this standard, the present work is a proof of principle, a necessary first step before committing time and expense to a more rigorous experiment.

In the next section, I review relevant theory from Reference [2], and recast it into a form better matched to the data analyzed here. In Section 3, I survey cloud chamber videos available online, explain why I chose the particular video analyzed here, and list the video's relevant technical specifications. In section 4, I explain how I extracted track coordinates, and I graph the distribution of coordinates for direct comparison with theory. Section 5 contains some concluding remarks.

\section{Theory}

Reference [2] addresses slow decays in which one heavy particle transforms into another by emitting a single, spinless nonrelativistic light charged particle. It argues that the probability per unit time and unit volume to find an emitted-particle track originating at position $\mathbf{x}$ and time $t$ is approximately

$$
\mathrm{P}(\mathbf{x}, \mathrm{t}) \sim \rho A s \tau|\psi(\boldsymbol{x}, t)|^{2}
$$

where $\psi$ is the emitted-particle wavefunction, $\rho$ and $\tau$ are constants characteristic of the cloud chamber medium, $s$ is a constant characteristic of the decay (emitted particle speed), and $A$ is a constant characteristic of ionizations triggered by emitted particles. For a slow decay, for times longer than the time needed for an emitted particle to cross the cloud chamber, the wavefunction within the cloud chamber takes the form

$$
\psi(x, t) \sim-i \mathrm{Y}(\Omega) \sqrt{\frac{\gamma}{s}} \frac{1}{r} \exp \left\{\left(\frac{r}{s}-t\right)\left(\frac{\gamma}{2}-i \frac{p s}{\hbar}\right)\right\}
$$

where $\mathrm{Y}$ is a spherical harmonic, $\Omega$ is solid angle, $r$ is distance from the initial heavy particle, $p$ is the magnitude of the emitted particle's momentum, and $\gamma$ is the conventional decay e-folding rate. Below, we treat $\mathrm{Y}$ as constant because single-particle decays detected in this paper's data are swave. It follows that for small $\gamma$ (slow decays), the number density depends on position close to the decay source according to

$$
N(\boldsymbol{x}) \propto \frac{1}{r^{2}}
$$

In the scenario highlighted in the next section, the cloud chamber is a thin flat circular enclosure (Petri dish) of height $a$, viewed from above. The radioactive sample is propped somewhere between the floor and roof of the chamber, so the two-dimensional density one actually observes is a sum or difference of two integrals over vertical column depth $z$, each of the following form, 


$$
D(x, y) \propto \int_{0}^{b}\left(\frac{1}{r^{2}+z^{2}}\right) d z
$$

where now $r$ is two-dimensional distance from the decay source, and $b$ is vertical distance from either bottom or top of the cloud chamber's active layer (sum if the radioactive source is inside the layer, difference otherwise). As a practical matter, we won't have enough statistics to do a good job measuring $D$ as a function of $x$ and $y$, so we'll default to the cumulative distribution as a function of $R$ :

$$
C(R) \propto \int_{0}^{R} r d r \int_{0}^{b}\left(\frac{1}{r^{2}+z^{2}}\right) d z \propto \ln \left[1+\left(\frac{R}{b}\right)^{2}\right]+\left(\frac{R}{b}\right)\left[\pi+2 \arctan \left(\frac{R}{b}\right)\right] \equiv c(R / b)
$$

If the source is equidistant between floor and roof of the Petri dish, and the entire dish is active for making tracks, then the cumulative radial distribution reduces to a single instance of Equation (1.5), with $b=a / 2$. If the source is propped against either floor or ceiling and the entire dish is active, then the cumulative radial distribution also reduces to a single instance of Equation (1.5), but with $b=a$.

\section{Data}

A Google search with terms "cloud chamber video" produces at least the twenty-three distinct examples in References [3-25]. Many of these don't apply here. References [3-12] show cloud chamber activity only from background radiation in the ambient environment. References [13, $14]$ provide clips of only a few seconds each. References $[15,16]$ show tracks from thorium rods, not point sources, and with uncontrolled viewing geometries. References [17-20] show tracks from lumps of material, making it impossible to look at small $R$. Also, by virtue of their nontrivial masses, they produce so many tracks that it's difficult to separate one track from another, even running the videos in slow motion. In Reference [21] the lump is quite small, but the cloud chamber track footage is very brief. References [22-25] all use small samples mounted at the ends of needles, i.e. the right physical geometry and nicely separable tracks. But Reference [22] shows only $8 \mathrm{sec}$ of track activity, with an enclosure of indeterminate size. Reference [23] also involves an enclosure of indeterminate size, as well as a variety of camera angles that complicate geometrical viewing analysis. Reference [24] has the best image quality, but uses three needles at the same time, so it's difficult to determine which track comes from which needle. Reference [25] seems to be the closest to "just right:" the enclosure is a Petri dish, whose dimensions are standardized, and the camera is located directly above (more or less) and fixed throughout the observation, which lasts a full $1 \mathrm{~min} 30 \mathrm{sec}$.

Detailed experimental specifications and caveats for Reference [25] are as follows:

- Chamber dimensions (presumed standard Petri dish): diameter 100mm, height $15 \mathrm{~mm}$.

- Radioactive source: labeled ${ }^{210} \mathrm{~Pb}$, but, because of decay chain, admixed with ${ }^{210} \mathrm{Bi}$ and ${ }^{210} \mathrm{Po}$. ${ }^{210} \mathrm{~Pb}$ and ${ }^{210} \mathrm{Bi}$ are beta emitters (half-lives 22.3 years and 5.0 days, respectively), and ${ }^{210} \mathrm{Po}$ is an s-wave alpha emitter (138 days) [26]. 
- Radioactive source fixture: eye of a needle, whose other end is stuck in a cork. (The radioactive region is about $4 \mathrm{~mm}$ long [R. Stevens, Spectrum Techniques Inc., private communication].) The entire assembly, including cork, is enclosed in the Petri dish.

- Location of radioactive source: xy coordinate of radioactive source not quite centered in Petri dish; vertical placement (in $z$ ) is unspecified, but data analysis (see below) suggests source is propped against roof of dish.

- Alcohol concentration: uncontrolled.

- Chamber temperature: uncontrolled; cooled with dry ice below.

- Source and fixture thermal characteristics: Indeterminate.

- Illumination: flashlight, handheld from the side, angle and brightness variable. Shadow of needle against dish floor is clearly visible, and complicates track measurement in its vicinity.

- Video camera location: Fixed, directly over top of Petri dish, pointing approximately straight down.

- Frame rate: 30fps; when downloaded to Microsoft Video Editor, frames are labeled minute:second:hundredth, and the hundredth is always of the form multiple-of-10 plus either 0,3 or 6 . Presumably 3 and 6 are rounded from 3.33 and 6.66 .

- Average rate of new track formation (see below): $2.5 / \mathrm{sec}$.

- First frame with cloud chamber tracks: 3:15:03, frame \#5852.

- Last frame with cloud chamber tracks: 4:45:46, frame \#8565.

- Notable anomalies: Tracks once formed seem to drift in a clockwise rotation.

\section{Measurement}

I downloaded the video of Reference [25] as an mp4 file onto a Dell laptop with screen resolution 1366x768, and manually stepped through frames 5852-8565 using Microsoft Video Editor in full-screen mode. Knowing the actual Petri dish dimensions, I was able to calibrate $0.16 \mathrm{~mm} / \mathrm{pixel}$. Every time I encountered a new track that appeared to point away from the source, I put the cursor over what I thought was the track's likely starting point, left-clicked, and read out the click pixel coordinates using the application "MacroRecorder," [https://www.macrorecorder.com/] (in the y direction, pixels count from the top). I stored the coordinates in the first tab of a spreadsheet (see Supplemental Material at [URL to be furnished] for spreadsheet contents). For every new track, the spreadsheet shows its video time, frame number, starting-point pixel coordinates, starting-point xy coordinates in $\mathrm{mm}$ relative to the radioactive source, and two-dimensional distance $R$ from the source divided by nominal vertical distance $b$ to active layer top or bottom. Each track is labeled short/long and diffuse/sharp based on eyeball judgements, as an aid to independent auditing. All tracks visible in this video are attributable to alpha particles (R. Schumacher, Carnegie Mellon University, private communication). A sample video frame is shown in Figure 1. 


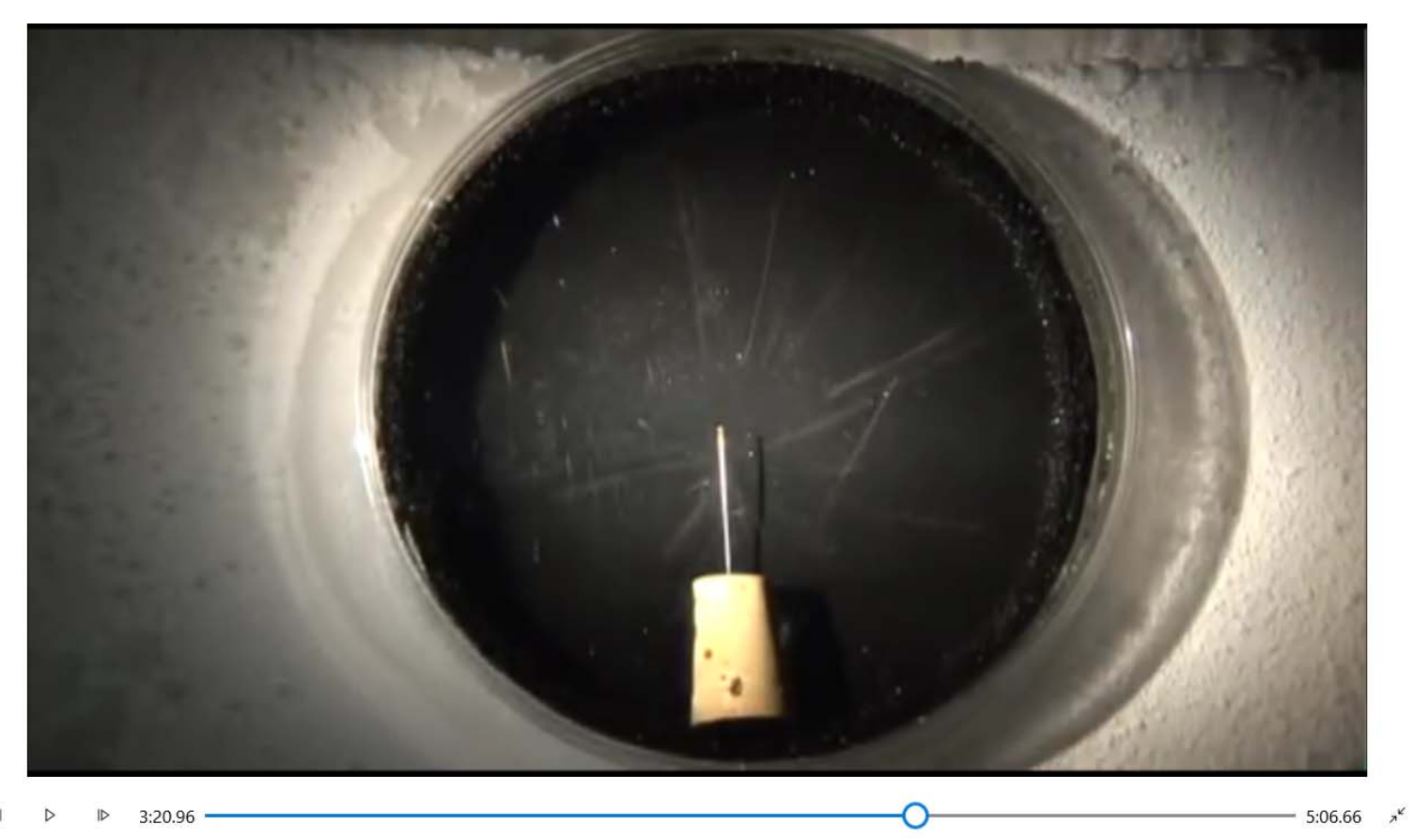

Figure 1: Frame \#6030 from Reference [25].

Readers are invited to check track identification directly against the actual video. It will be seen that the first appearance of a track is often difficult to discern, because tracks start faint and then "develop" over time as their constituent droplets expand. When stepping through the video, it may be necessary to look over a short time period past the frame at which a new track is indicated, to see that the faint smudge at that location in that frame will in fact develop into a track. The reader will also see that track-origin identification is often made difficult and even ambiguous by the non-ideal placement of camera and illumination source.

In principle one must distinguish between alpha and beta tracks because the theory in Section 2 applies to the alpha emitter ${ }^{210} \mathrm{Po}$ but not to the beta emitters ${ }^{210} \mathrm{~Pb}$ and ${ }^{210} \mathrm{Bi}$. Since beta decay also involves a neutrino, it is emission of two particles with an energy spectrum for the outgoing charged particle, rather than emission of a single charged particle with a unique outgoing energy. However, beta tracks are too tenuous to be seen in this video (R. Schumacher, Carnegie Mellon University, private communication).

To compare with the theory in Section 2, I first put together a cumulative distribution. I sort the spreadsheet on the values of $R / b$ for alpha tracks, then number the values starting at the smallest, and finally graph the pairs $[R / b$, sort index $]$ on a scatter plot. I compare this directly with a vertically scaled graph of $c(R / b)$ for Equation (1.5). This comparison is shown in Figure 2. We can already tell from this figure that the radioactive sample is probably situated inside or extremely close to the track-active layer, because otherwise, following Equation (1.4), the blue curve should look strongly quadratic near $R=0$. I have chosen the value of $b$ that gives the best match for $d(R / b=1) / d(R / b=0.5)$, where $d$ is the cumulative radial distribution. That turns out be $b \sim 15 \mathrm{~mm}$, 
suggesting the radioactive source is pinned against the roof of the Petri dish. I have vertically scaled the $c(R / b)$ points so they match the measured points at $R / b=0.5$.

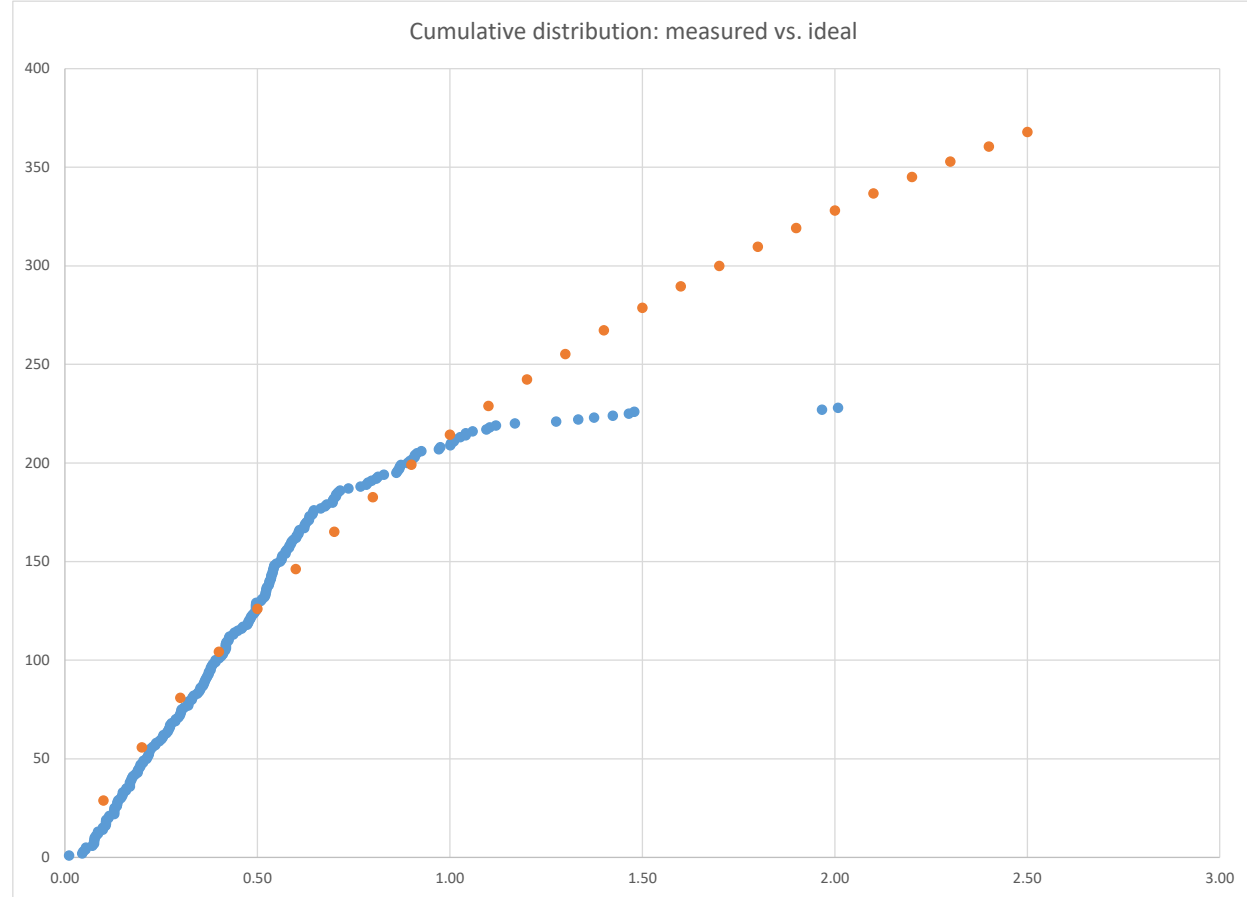

Figure 2: Measured (blue) vs. ideal (red) cumulative distribution (number of counts), as functions of $R / b . b$ is set so the two data sets have the same ratio of value at $R / b=1.0$ to value at $R / b=0.5$. Vertical scale of red points $[c(R / b)]$ is set so that the two data sets match at $R / b=0.5$

The two data sets (measured and scaled ideal) line up, more or less, until the measured curve appears to turn over and prematurely flatten around $R / b=1.0(R=15 \mathrm{~mm})$. This means a shortfall in observed detections that originate at - rather than just pass through - large $R$. (According to Reference [2], track origination requires the presence of a vapor droplet that is sub-critical but just barely so; track continuation does not). This could mean the theory in Reference [2] is wrong. Or the lack of track originations at large $R>15 \mathrm{~mm}$ could be a temperature periphery effect: Maybe the Petri dish is warmer at its extremities, making it harder for subcritical vapor droplets to form spontaneously by thermal fluctuation. But this is not likely, because the volume outside $R=15 \mathrm{~mm}$ is about $90 \%$ of the entire Petri dish, straining the concept of "extremity." Perhaps the lack of track originations at $R=15 \mathrm{~mm}$ is actually a breakdown of the Born rule within the framework of Reference [2]: Maybe, beyond $R=15 \mathrm{~mm}$, the alpha wavefunction in Equation (1.2) is so attenuated that the chamber simply runs out of subcritical droplets close enough to critical for such a weak wavefunction to push into visibility. [For reference, the value of $\mid$ wavefunction $\left.\right|^{2}$ at $R=15 \mathrm{~mm}$, according to Equation (1.2), and ignoring the exponential factor and using $Y=1 /(4 \pi)^{1 / 2}$, is roughly $10^{-12} \mathrm{~m}^{-3}$.] One way to test this would be to use an alternative alpha emitter with roughly $1 / 10$ the half-life of ${ }^{210} \mathrm{Po}$. Then the factor $\gamma$ in Equation 1.2 would guarantee that the wavefunction wouldn't get weak enough to fail to start tracks until $R$ increased by a factor of $\sim 3$, from $15 \mathrm{~mm}$ to the full $50 \mathrm{~mm}$ radius of the dish, and then track originations would be seen throughout the chamber. (This assumes that $t$ in Equation (1.2) is small enough that the factor $\mathrm{e}^{-\gamma t}$ is $\mathrm{O}(1)$ for both 
the ${ }^{210} \mathrm{Po}$ of Reference [25] and the hypothetical alternative alpha emitter.) This finding suggests modifying the Born rule as shown schematically in Figure 3. In this modification concept, the cutoff value of $|\psi|^{2}$ depends on the details of the measuring apparatus. One might think of this as modifying quantum efficiency by a wavefunction-dependent factor that is unity when wavefunction is sufficiently large, and zero when sufficiently small. Conceivably, this could lead certain experiments to overestimate the lifetimes of extremely rare decays.

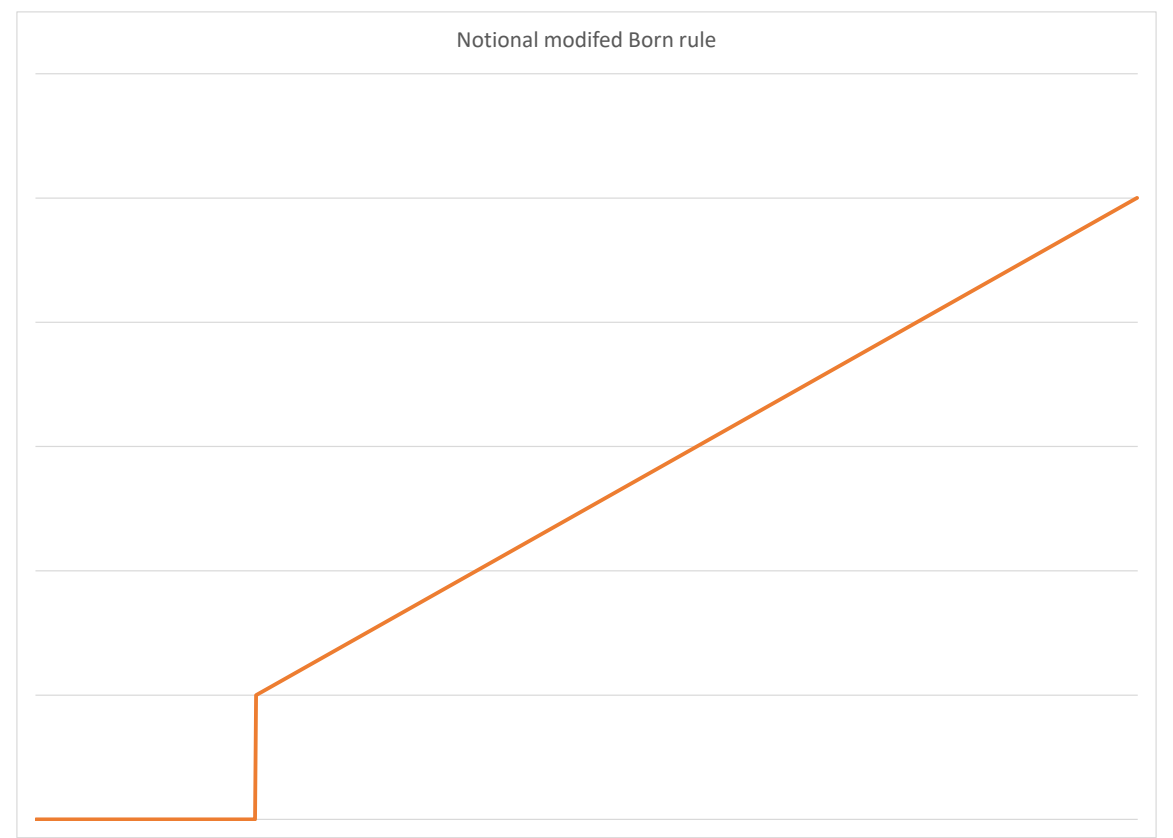

Figure 3: Notional modification of Born rule. Horizontal axis represents $\mid$ wavefunction $\left.\right|^{2}$ and vertical axis represents probability density. Units are arbitrary. The wavefunction value at the cutoff would depend on particulars of the measurement system.

One might have expected a track-origination deficit at small $R$ because the radioactive source generates heat that can suppress vapor supersaturation. That's not seen in Figure 2, presumably because the source is so small.

\section{Concluding remarks}

Using opportunistic data, I have carried out a proof of principle for a previously unappreciated, low-cost probe of quantum mechanics fundamentals. It is also a novel tabletop example of a wavefunction with a signature visible to the naked eye without cryogenic temperatures (e.g., superconductivity) or micron-scale detectors and finely controlled coherence (e.g., double-slit interference). The data in this paper may already show violation of Born's rule.

The ideal experiment tailored to this science would address the caveats identified in Section 3 . It would also include the following improvements:

- Several interchangeable alpha emitters with half-lives spread over an order of magnitude or more.

- Automated track detection and classification, to eliminate reliance on eyeball judgement.

- Track detection in 3D, and sufficient statistics to measure three-dimensional distributions. 
- Sufficient control and instrumentation to see deviations from Born rule (if we have not seen them here already for $R>15 \mathrm{~mm}$ ).

- Sufficient instrumentation to probe dynamics of individual droplet formation.

\section{Acknowledgements}

I am grateful to Steve Gagnon, Reinhard Schumacher, Roger Stevens and Frank Taylor for helpful correspondence.

\section{Author contributions}

The single author of this paper is solely responsible for its content.

\section{Competing interests}

The author declares no competing interests.

\section{References}

1. Allahverdyan, A. E., Balian, R., Nieuwenhuizen, T. M. Understanding quantum measurement from the solution of dynamical models. Physics Reports. 2013; 525: 1-166.

2. Schonfeld, J. F. The first droplet in a cloud chamber track. Foundations of Physics. 2021; 51: 47.

3. https://www.youtube.com/watch? $\mathrm{v}=\mathrm{i} 15 \mathrm{ef618DP0}$ Nuledo, 8/14/2018, ambient

4. https://www.youtube.com/watch? $v=$ jh7SzrNWGhI Exploratorium, 6/7/2021, ambient

5. https://www.youtube.com/watch?v=eh3bxXHqF2U CuriosityStream, 11/14/2019, ambient

6. https://www.facebook.com/TRIUMFLab/videos/cloud-chambervideo/2447281728728624/?extid=SEO---- TRIUMF, 12/17/2019, ambient

7. https://techtv.mit.edu/videos/3141-cloud-chamber MIT, 4/12/2018, ambient

8. https://videos.cern.ch/record/2729964 CERN, 9/8/2020, ambient

9. https://mediaspace.nau.edu/media/What+Radon+Looks+Like+in+a+Cloud+Chamber/0_vcb4 opj0/69042112 Northern Arizona University, 4/11/2017, ambient

10. https://www.sciencephoto.com/media/681631/view/cloud-chamber SciencePhotoLibrary, ambient

11. http:// en.wikipedia.org/wiki/File:Cloud chamber.ogg Wikipedia, 12/15/2009, ambient

12. https://www.shutterstock.com/video/clip-4059667-radioactive-particle-cloud-chamber Shutterstock, ambient

13. https://www.cloudchambers.com/videodownloads.htm few-second clips

14. https://www.youtube.com/watch? $\mathrm{v}=\mathrm{uRN} 09 \mathrm{fXA3Mw}$ IOP, 7/24/2019, source indeterminate

15. https://www.youtube.com/watch?v=e3fi6uyyrEs Harvard, 3/6/2014, thorium rod

16. https://www.esa.int/ESA Multimedia/Videos/2014/07/Cloud chamber classroom_demonstration_video_VP03 ESA, 7/30/2014, thorium rods

17. https://www.youtube.com/watch?v=ZiscokCGOhs Cloudylabs, 1/2/2014, pitchblende lump

18. https://www.youtube.com/watch?v=NeydrHKvpYM American Nuclear Society, 8/9/2007, uranite lump.

19. https://www.youtube.com/watch?v=noP7HT-Uins Alejandro del Mazo Vivar, 12/16/2013, $\mathrm{Am}^{241}$ lump, expansion chamber, one megaburst at a time. 
20. https://www.youtube.com/watch?v=u4UoCWpuEvg QuantumBoffin, 3/22/2014, ${ }^{239} \mathrm{Pu}$ and ${ }^{90} \mathrm{Sr}$ lumps.

21. https://www.youtube.com/watch?v=xwYSvzojFbo IOP, 7/24/2019, americium source, not point-like but very small.

22. https://www.youtube.com/watch? $\mathrm{v}=\operatorname{chxv5G6UF10}$ CosmoLearning, 1/1/2008, indeterminate needle source

23. https://www.youtube.com/watch?v=AzMi2AtHh5k SUNY Geneseo, 1/31/2018, ${ }^{210} \mathrm{~Pb}$ needle source (https://www.geneseo.edu/fletcher/cloud_chamber).

24. https://www.youtube.com/watch?v=LosCtIh5Flc Carnegie Mellon University, 7/23/2017, three simultaneous needle sources.

25. https://www.youtube.com/watch?v=pewTySxfTQk Jefferson Lab, 10/25/2010, ${ }^{210} \mathrm{~Pb}$ needle source.

26. http://nucleardata.nuclear.lu.se/toi/nuclide.asp?iZA=820210, http://nucleardata.nuclear.lu.se/toi/nuclide.asp?iZA=830210, http://nucleardata.nuclear.lu.se/toi/nuclide.asp?iZA=840210 


\section{Supplementary Files}

This is a list of supplementary files associated with this preprint. Click to download.

- Framecounter090921tab1.pdf

- Framecounter090921tab2.pdf

- Framecounter090921tab3.pdf 